\title{
Etomidato e vecurônio na indução anestésica de cardiopatas chagásicos crônicos
}

\author{
Etomidate and vecuronium in induction of anesthesia of \\ chronic chagasic cardiac patients \\ Nilson de Camargos Roso, João Abrão e José Álves Neto
}

\begin{abstract}
Resumo Com o objetivo de avaliar as respostas hemodinâmicas e cardiovasculares durante a indução anestésica com etomidato (hipnótico) e vecurônio (bloqueador neuromuscular) na doença de Chagas humana analisaram-se 41 pacientes (15 chagásicos e 26 não chagásicos). Durante $o$ ato anestésico colheu-se sangue para sorologia e foram registrados pressões arteriais, freqüência e ritmos cardíacos e saturação arterial de oxigênio em seis momentos diferentes. A análise das pressões arteriais e da freqüência cardíaca, tanto nos chagásicos como nos não chagásicos, mostrou variação significante nos diferentes tempos do ato anestésico, mas não entre os dois grupos no mesmo tempo. A saturação arterial de oxigênio manteve-se constante em todos os casos estudados. Conclui-se que as duas drogas são seguras para uso na indução da anestesia do chagásico crônico.
\end{abstract}

Palavras-chaves: Doença de Chagas. Anestesia geral. Etomidato. Brometo de Vecurônio. Hemodinâmica.

\begin{abstract}
Fifteen chagasic and 26 non-chagasic patients were evaluated for hemodynamic and cardiovascular responses during induction of anesthesia with etomidate (a hypnotic agent) and Vecuronium (a neuromuscular blocker). Blood for serum testing was collected during anesthesia. Blood pressure, heart rate and rhythm, mean arterial pressure and arterial oxygen saturation were also monitored on six different occasions during anesthesia. When the various stages of anesthesia were compared, significant differences in blood pressure and heart rate were observed. For any of the given stages of anesthesia blood pressure and heart rate did not show significant differences when chagasic were compared to non-chagasic patients. Arterial oxygen saturation remained steady in all cases. We conclude that both drugs are safe for use during the induction of anesthesia in chronic chagasic patients.
\end{abstract}

Key-words: Chagas' disease. Anesthesia general. Etomidate. Vecuronium bromide. Hemodynamic.

Para os anestesiologistas, dentre as várias formas anatomoclínicas da doença de Chagas (DC), têm maior importância aquelas que acometem, de maneira mais intensa, o coração, já que as drogas utilizadas em anestesia, na sua maioria, atuam como depressoras da função cardíaca1 12.
Em cardiopatas não chagásicos, há vários anos, na indução da anestesia, vem se utilizando o etomidato (hipnótico) e o vecurônio (bloqueador neuromuscular), com resultados bastante satisfatórios 7910 . Outras drogas que não estas podem levar a indesejáveis complicações3 4589 .

\footnotetext{
Disciplina de Anestesiologia da Faculdade de Medicina do Triângulo Mineiro. Uberaba, MG. Apoio financeiro: Fundação de Amparo à Pesquisa do Estado de Minas Gerais (FAPEMIG). Endereço para correspondência: Dr. Nilson de Camargos Roso. Alameda Real 400, 38057-010 Uberaba, MG, Brasil. Fax: 5534 318-5269

Recebido para publicação em 5/1/98.
} 
Não havendo estudos sistematizados sobre o uso do etomidato e do vecurônio em portadores da tripanosomose cruzi, nos pareceu

anestésico.

\section{MATERIAL E MÉTODOS}

O material consta de 41 pacientes, 16 homens e 25 mulheres, submetidos a anestesia geral no Hospital Escola da Faculdade de Medicina do Triângulo Mineiro (HE/FMTM), divididos em dois grupos.

Grupo l: 15 chagásicos crônicos, 7 homens e 8 mulheres, com idade variando de 39 a 74 anos (55 $\pm 12,21)$, dos quais $12 \mathrm{com}$ a forma cardíaca e $3 \mathrm{com}$ a forma mista (cardíaca e digestiva) da doença.

Grupo II (controle): 26 pacientes, não chagásicos, sendo 9 homens e 17 mulheres, com idade entre 17 a 63 anos $(39 \pm 12,66)$

O diagnóstico de DC foi estabelecido pela positividade no sangue, de pelo menos dois dos seguintes testes: hemaglutinação indireta, imunofluorescência indireta e imunoenzimático (ELISA). A determinação da forma anatomoclínica dos chagásicos foi baseada nos dados clínicos, no eletrocardiograma convencional e no estudo radiológico do coração, esôfago e/ou colon.

Nos 3 chagásicos com a forma mista da doença a indicação cirúrgica em 2 foi para tratamento de megaesôfago e em 1 para megacólon. Nos 12 cardiopatas chagásicos crônicos as cirurgias foram eletivas, por motivos não relacionados à tripanosomíase (colecistectomia, tireoidectomia, mamoplastia redutora, nefrectomia, etc.). Os 26 não chagásicos, não tinham sinais e sintomas sugestivos de cardiopatia e foram submetidos a cirurgias eletivas.

Todos os pacientes pertenciam, quanto ao estado físico, às classes I ou II do critério de avaliação usado pela Sociedade Americana de Anestesiologia (ASA) 12 .

Após aprovação do projeto pelo Conselho de Ética Médica do HE/FMTM e consentimento verbal dos pacientes, foi iniciado o trabalho que pertinente avaliar as respostas hemodinâmicas e cardiovasculares em cardiopatas chagásicos crônicos durante o ato

se desenvolveu durante os anos de 1995 e 1996. $\mathrm{O}$ ato anestésico foi sempre de responsabilidade de pelo menos um dos autores deste trabalho.

Todos os pacientes foram anestesiados com o seguinte protocolo: pré-medicação na sala de cirurgia com diazepam ( $5 \mathrm{mg}$ IV) e fentanil $(2 \mu \mathrm{g} / \mathrm{kg} \mathrm{IV})$, desnitrogenação por seis minutos, indução da anestesia venosa com etomidato $(0,3 \mathrm{mg} / \mathrm{kg})$ e vecurônio $(0,1 \mathrm{mg} / \mathrm{kg})$. Ventilava-se por dois minutos enquanto se aguardava o relaxamento adequado para se realizar a intubação orotraqueal sob visão direta. $\mathrm{Na}$ manutenção da anestesia usou-se o enflurano. Os anestesiologistas envolvidos no estudo, durante o ato anestésico, não tinham conhecimento do grupo a que pertenciam os doentes.

Os parâmetros avaliados foram: pressão arterial sistólica (PAS), pressão arterial diastólica (PAD), pressão arterial média (PAM), freqüência cardíaca (FC), ritmo cardíaco e saturação arterial de oxigênio $\left(\mathrm{SaO}_{2}\right)$. Os três primeiros foram registrados com o auxílio de um aparelho automático de medida da pressão arterial não invasivo marca Dixtal, modelo DX2710 e um eletrocardiógrafo marca FUNBEC, modelo 4F. A $\mathrm{SaO}_{2}$ foi medida com um oxímetro de pulso da marca Ohmeda modelo DIOXI 3700.

Os registros da PAS, PAD, PAM, FC, $\mathrm{SaO}_{2} \mathrm{e}$ do eletrocardiograma (ECG) foram feitos em momentos pré-estabelecidos: T1 (paciente na sala de cirurgia, após pré-medicação), T2 (imediatamente após a injeção das drogas de indução), T3 (imediatamente após a intubação orotraqueal), T4 (1 minuto após T3), T5 ( 1 minuto após T4), T6 (1 minuto após T5).

O estudo estatístico constou de análise do comportamento de cada variável no seu grupo e em cada momento o valor desta variável foi comparado com o do grupo controle. Para tal foram empregados a análise da variância para medidas repetidas e o teste $t$ de Student para

comparação de duas médias. A homogeneidade da variância foi analisada pelo teste de Bartlett. O

nível de significância foi fixado em $5 \%$ ( $p<$ $0,05)$.

\section{RESULTADOS}

As pressões arteriais médias e a freqüência 
Revista da Sociedade Brasileira de Medicina Tropical 32:41-46, jan-fev, 1999.

\begin{tabular}{|c|c|c|c|}
\hline Número do caso & PAM1 & PAM2 & PAM3 \\
\hline \multicolumn{4}{|l|}{ Chagásicos } \\
\hline 1 & 134 & 95 & 195 \\
\hline 2 & 110 & 95 & 119 \\
\hline 3 & 106 & 79 & 95 \\
\hline 4 & 80 & 82 & 113 \\
\hline 5 & 96 & 97 & 113 \\
\hline 6 & 78 & 109 & 93 \\
\hline 7 & 140 & 107 & 145 \\
\hline 8 & 117 & 69 & 163 \\
\hline 9 & 100 & 71 & 77 \\
\hline 10 & 156 & 120 & 148 \\
\hline 11 & 107 & 91 & 108 \\
\hline 12 & 110 & 83 & 131 \\
\hline 13 & 110 & 105 & 87 \\
\hline 14 & 97 & 88 & 114 \\
\hline 15 & 127 & 132 & 150 \\
\hline \multicolumn{4}{|l|}{ Não chagásicos } \\
\hline 16 & 121 & 93 & 149 \\
\hline 17 & 94 & 116 & 94 \\
\hline 18 & 109 & 79 & 102 \\
\hline 19 & 113 & 90 & 121 \\
\hline 20 & 112 & 111 & 121 \\
\hline 21 & 80 & 75 & 86 \\
\hline 22 & 76 & 69 & 91 \\
\hline 23 & 90 & 86 & 112 \\
\hline 24 & 83 & 85 & 99 \\
\hline 25 & 119 & 84 & 123 \\
\hline 26 & 92 & 78 & 118 \\
\hline 27 & 100 & 87 & 136 \\
\hline 28 & 92 & 106 & 116 \\
\hline 29 & 99 & 82 & 88 \\
\hline 30 & 120 & 116 & 148 \\
\hline 31 & 100 & 64 & 107 \\
\hline 32 & 110 & 140 & 140 \\
\hline 33 & 92 & 99 & 129 \\
\hline 34 & 147 & 90 & 158 \\
\hline 35 & 88 & 110 & 99 \\
\hline 36 & 78 & 70 & 114 \\
\hline 37 & 93 & 87 & 88 \\
\hline 38 & 133 & 103 & 149 \\
\hline 39 & 90 & 77 & 103 \\
\hline 40 & 90 & 100 & 110 \\
\hline 41 & 102 & 101 & 112 \\
\hline
\end{tabular}




\begin{tabular}{|c|c|c|c|}
\hline Número do caso & FC1 & FC2 & FC3 \\
\hline \multicolumn{4}{|l|}{ Chagásicos } \\
\hline 1 & 97 & 100 & 100 \\
\hline 2 & 60 & 60 & 60 \\
\hline 3 & 72 & 71 & 85 \\
\hline 4 & 65 & 64 & 90 \\
\hline 5 & 107 & 96 & 110 \\
\hline 6 & 106 & 108 & 105 \\
\hline 7 & 102 & 77 & 96 \\
\hline 8 & 62 & 71 & 92 \\
\hline 9 & 72 & 67 & 75 \\
\hline 10 & 66 & 53 & 88 \\
\hline 11 & 86 & 89 & 93 \\
\hline 12 & 107 & 98 & 110 \\
\hline 13 & 66 & 76 & 69 \\
\hline 14 & 61 & 58 & 92 \\
\hline 15 & 70 & 137 & 100 \\
\hline \multicolumn{4}{|l|}{ Não chagásicos } \\
\hline 16 & 78 & 78 & 76 \\
\hline 17 & 72 & 100 & 83 \\
\hline 18 & 87 & 69 & 81 \\
\hline 19 & 58 & 49 & 66 \\
\hline 20 & 60 & 56 & 58 \\
\hline 21 & 67 & 65 & 97 \\
\hline 22 & 65 & 62 & 68 \\
\hline 23 & 100 & 86 & 89 \\
\hline 24 & 90 & 78 & 54 \\
\hline 25 & 70 & 62 & 97 \\
\hline 26 & 72 & 83 & 74 \\
\hline 27 & 94 & 92 & 107 \\
\hline 28 & 83 & 64 & 100 \\
\hline 29 & 89 & 70 & 72 \\
\hline 30 & 52 & 61 & 108 \\
\hline 31 & 72 & 61 & 70 \\
\hline 32 & 102 & 114 & 120 \\
\hline 33 & 81 & 71 & 101 \\
\hline 34 & 98 & 90 & 110 \\
\hline 35 & 82 & 88 & 87 \\
\hline 36 & 85 & 86 & 78 \\
\hline 37 & 81 & 84 & 90 \\
\hline 38 & 98 & 70 & 101 \\
\hline 39 & 78 & 81 & 99 \\
\hline 40 & 58 & 51 & 73 \\
\hline 41 & 74 & 82 & 92 \\
\hline
\end{tabular}

cardíaca, em cada grupo, variaram significantemente $(p<0,01)$ nos seis tempos considerados (Tabelas 1 e 2). Quando se compara os valores das pressões e da freqüência, nos dois grupos para um mesmo tempo, não se detecta variações significantes.

Sete (46,6\%) dos 15 chagásicos e 9 (34,6\%) dos 26 não chagásicos apresentaram níveis de PAD igual ou maior que $90 \mathrm{mmHg}$ (Tabela 3).
Freqüência cardíaca superior a $80 \mathrm{bpm}$ foi detectada em 6 (40\%) dos chagásicos e em 13 (50\%) dos não chagásicos.

A $\mathrm{SaO}_{2}$ manteve-se constante tanto nos chagásicos como nos não chagásicos.

Em dois chagásicos foram detectadas, nos momentos T3 e T4, alterações do ritmo cardíaco: um desenvolveu extra-sístoles ventriculares e 
Tabela 3 - Médias e desvios padrões das pressões arteriais sistólica, diastólica, média e freqüência cardíaca.

\begin{tabular}{|c|c|c|c|c|c|c|c|c|}
\hline \multirow{3}{*}{ Tempos } & \multicolumn{8}{|c|}{ Parâmetros hemodinâmicos } \\
\hline & \multicolumn{2}{|c|}{ PAS } & \multicolumn{2}{|c|}{ PAD } & \multicolumn{2}{|c|}{ PAM } & \multicolumn{2}{|c|}{ FC } \\
\hline & $\mathrm{Ch}$ & $\mathrm{NCh}$ & $\mathrm{Ch}$ & $\mathrm{NCh}$ & $\mathrm{Ch}$ & $\mathrm{NCh}$ & $\mathrm{Ch}$ & $\mathrm{NCh}$ \\
\hline \multirow[t]{2}{*}{$\overline{\mathrm{T} 1}$} & $142,80 \pm 26,98$ & $134,03 \pm 19,99$ & $89,60 \pm 14,09$ & $83,42 \pm 13,22$ & $111,29 \pm 22,05$ & $100,12 \pm 17,16$ & $79,93 \pm 18,66$ & $78,69 \pm 13,91$ \\
\hline & \multicolumn{2}{|c|}{$p=0,24$} & \multicolumn{2}{|c|}{$p=0,16$} & \multicolumn{2}{|c|}{$p=0,08$} & \multicolumn{2}{|c|}{$p=0,80$} \\
\hline \multirow[t]{2}{*}{ T2 } & $129,07 \pm 29,26$ & $125,23 \pm 21,30$ & $81,67 \pm 14,88$ & $78,65 \pm 15,27$ & $94,87 \pm 17,57$ & $92,23 \pm 17,48$ & $81,67 \pm 22,80$ & $75,12 \pm 15,472$ \\
\hline & \multicolumn{2}{|c|}{$p=0,63$} & \multicolumn{2}{|c|}{$p=0,55$} & \multicolumn{2}{|c|}{$p=0,65$} & \multicolumn{2}{|c|}{$p=0,27$} \\
\hline \multirow[t]{2}{*}{ T3 } & $160,60 \pm 26,09$ & $151,39 \pm 25,74$ & $112,80 \pm 25,06$ & $98,27 \pm 20,12$ & $123,40 \pm 31,96$ & $115,89 \pm 20,87$ & $93,13 \pm 11,43$ & $86,58 \pm 17,04$ \\
\hline & \multicolumn{2}{|c|}{$p=0,28$} & \multicolumn{2}{|c|}{$p=0,05^{*}$} & \multicolumn{2}{|c|}{$p=0,63$} & \multicolumn{2}{|c|}{$p=0,19$} \\
\hline \multirow[t]{2}{*}{ T4 } & $142,67 \pm 30,66$ & $136,35 \pm 26,29$ & $93,00 \pm 22,52$ & $87,42 \pm 20,28$ & $114,27 \pm 29,02$ & $102,04 \pm 24,11$ & $83,93 \pm 20,95$ & $81,58 \pm 19,01$ \\
\hline & \multicolumn{2}{|c|}{$\mathrm{p}=0,50$} & \multicolumn{2}{|c|}{$p=0,57$} & \multicolumn{2}{|c|}{$p=0,15$} & \multicolumn{2}{|c|}{$p=0,72$} \\
\hline \multirow[t]{2}{*}{ T5 } & $127,47 \pm 26,37$ & $124,35 \pm 22,77$ & $84,27 \pm 18,57$ & $83,35 \pm 29,15$ & $99,40 \pm 27,73$ & $93,62 \pm 19,84$ & $83,93 \pm 20,95$ & $81,58 \pm 19,01$ \\
\hline & \multicolumn{2}{|c|}{$p=0,69$} & \multicolumn{2}{|c|}{$p=0,91$} & \multicolumn{2}{|c|}{$p=0,55$} & \multicolumn{2}{|c|}{$p=0,72$} \\
\hline \multirow[t]{2}{*}{ T6 } & $117,87 \pm 24,05$ & $125,54 \pm 21,88$ & $76,27 \pm 13,08$ & $82,39 \pm 22,90$ & $89,27 \pm 15,36$ & $69 \pm 24,75$ & $81,00 \pm 24,97$ & $76,00 \pm 13,50$ \\
\hline & \multicolumn{2}{|c|}{$p=0,30$} & \multicolumn{2}{|c|}{$p=0,65$} & \multicolumn{2}{|c|}{$p=0,55$} & \multicolumn{2}{|c|}{$p=0,59$} \\
\hline
\end{tabular}

$\overline{\mathrm{PAS}}=$ pressão arterial sistólica; PAD = pressão arterial diastólica; $\mathrm{PAM}=$ pressão arterial média; $\mathrm{FC}=$ freqüência cardíaca; $\mathrm{T} 1=$ antes da pré-medicação \pm basal), T2 = após indução, T3 = após intubação orotraqueal, T4 = um minuto após T3, T5 = um minuto após T4, T6 = um minuto após T5; Ch = chagásicos, $\mathrm{NCh}=$ não chagásicos.

* diferenças significantes entre os dois grupos.

outro bradicardia importante $(<60 \mathrm{bpm})$, que cederam com tratamento específico (lidocaína e

atropina). Dois não chagásicos, no momento T3, apresentaram distúrbio do ritmo cardíaco: um desenvolveu bradicardia e o outro extra-sístole ventricular.

\section{DISCUSSÃO}

A análise da Tabela 3 mostra uma única diferença estatisticamente significante, entre chagásicos e não chagásicos: a PAD no momento T3 em relação a T2. Este achado poderia ser atribuído ao estresse da laringoscopia e da intubação orotraqueal, como já demonstrado em não chagásicos6, mas não justifica a razão da resposta ser maior nos chagásicos do que nos não chagásicos em T3.

A predominância de hipertensos no grupo chagásico poderia sugerir que a hipertensão arterial sistêmica tenha contribuído para a resposta diferenciada observada. A favor desta hipótese há o fato dos hipertensos, quando submetidos à anestesia, apresentarem maior labilidade pressória13. Nossos dados não permitem esclarecer a questão e novos estudos deverão ser feitos sobre o assunto. Freqüência cardíaca superior a $80 \mathrm{bpm}$ e alterações do ritmo cardíaco foram observados nos dois grupos, não influenciando nos resultados. Associando-se estes dados aos outros achados do presente estudo, somos levados a admitir que também na indução anestésica de cardiopatas chagásicos deve-se optar pelo emprego de etomidato e de vecurônio.

Menção à parte, merece o chagásico classificado na forma indeterminada da doença. Nesta, os pacientes são assintomáticos e têm exames clínico, radiológicos (coração, esôfago e cólons) e eletrocardiográfico convencional, normais ${ }^{11}$. Ainda que os chagásicos incluídos nesta forma da enfermidade, quando submetidos a exames mais sensíveis possam revelar alterações², a qualidade e intensidade destas não

parecem suficientes para possibilitar uma resposta que contra-indique o emprego do etomidato e vecurônio. Não temos elementos para opinar sobre o emprego das referidas drogas nos chagásicos na fase aguda.

Em conclusão parece-nos recomendável o emprego de etomidato e vecurônio na indução da anestesia geral em chagásicos crônicos

\section{AGRADECIMENTOS}

Aos Professores Edison Reis Lopes e Aluízio

Prata, pelo incentivo e pelas valiosas sugestões na elaboração do texto. 
1. Barbosa JS, Saraiva RA, Vieira ZEG. Efeitos dos anestésicos no cardiopata chagásico. Revista Brasileira de Anestesiologia 34:159-64, 1984.

2. Barreto ACP, Azul LGS, Mady C, lanni BM, Vianna CB. Belloti G, Pileggi F. Forma indeterminada da doença de Chagas. Uma doença polimórfica. Arquivos Brasileiros de Cardiologia 55:347-353, 1990.

3. Buljubasic N, Marijic J, Berczi V, Supan DF, Kampine JP, Bosnjak ZJ. Differencial effects of etomidate, propofol, and midazolam on calcium and potassium channel currents in canine myocardial cells. Anesthesiology 85:1092-1099, 1996.

4. Chari P, Grover VK, Chawla S, Gujral JS. Cardio-vascular effects of vecuronium in patients undergoing elective closed mitral valvotomy. Indian Journal of Medical Research 94:211-216, 1991.

5. Eames WO, Rooke GA, Wu RS, Bishop MJ. Comparison of the effects of etomidate, propofol, and thiopental on respiratory resistance after tracheal intubation. Anesthesiology 84:1307-1311, 1996.

6. Fox EJ, Sklar GS, Hill CH, Villanueva R, King BD.
Complications related to the pressor response to endotracheal intubation. Anesthesiology 47:524-525, 1977.

7. Gallo JA, Cork RC, Puchi P. Comparison of effects of atracurium and vecuronium in cardiac surgical patients. Anesthesia and Analgesia 67:161-165, 1988.

8. Jellish WS, Riche H, Salord F, Ravussin P, Tempelhoff R. Etomidate and thiopental-based anesthetic induction: comparisons between different titrated levels of electrophysiologic cortical depression and response to laryngoscopy. Journal of Clinical Anesthesia 9:36-41, 1997.

9. Mazerolles M, Senard JM, Verwaerde P, Tran MA, Montastruc JL, Virenque C, Montastruc P. Effects of pentobarbital and etomidadte on plasma catecholamine levels and spectral analysis of blood pressure and heart rate in dogs. Fundaments of Clinical Pharmacology 10:298-303, 1996.

10. Owen $H$, Spence AA. Etomidate. British Journal of Anaesthesia 56:556-557, 1984.

11. I Reunião de Pesquisa Aplicada em Doença de Chagas. 